\title{
Supporting personalized care of older adults with vision and cognitive impairments by user modeling
}

\author{
Petr Bilek, Miroslav Macik, Zdenek Mikovec \\ Department of Computer Graphics and Interaction \\ Faculty of Electrical Engineering, Czech Technical University in Prague \\ Karlovo nam. 13, Praha 2 \\ Email: macikmir@fel.cvut.cz
}

\begin{abstract}
We present a user modeling approach tailored to the user group of older adults with vision impairments. Our previous work with this user group has been followed by qualitative user research with the staff of a residential care facility for visually impaired older adults. We defined the structure of a user model that represents aspects related to personal psychological development and attributes that affect interaction with technologies. Furthermore, we present two generations of prototypes of administration UI for the user model. Results of the qualitative evaluation are discussed in the paper. This effort aims to help the personnel of specialized care institution in providing personalized care and make the interaction with technologies accessible for the group of visually impaired older adults with vision impairments.
\end{abstract}

\section{INTRODUCTION}

A CCORDING to [1] the prevalence of blindness is globally $0.48 \%$, and $2.95 \%$ of world population deals with moderate to severe vision impairment. The majority of visually impaired people appear among older adults, as $52.9 \%$ of visually impaired people are older than 70 years.

The proportion of older adults in the population is steadily rising. In addition, according to $\mathrm{WHO}$ this trend will continue (due to increasing life expectancy and decreasing birth rate) [2]. During ageing, a large proportion of the population is acquiring some kind of health impairment. This fact can be also seen in data of Czech statistical office (data were processed in 2013) [3], 18.6\% of people between the ages of 60 and 74 have some kind of disability. In category 75+ years old, percentage of disabled people rapidly rises, it is around $42.1 \%$.

Unfortunately, the research attention on older adults with vision impairments (VI) is limited. We have analyzed 39 papers focusing on people with VI presented on last three $\mathrm{CHI}$ conferences (2016-2018). When excluding studies focused mainly on children and young adults, the average age of study participant was 37.3 years (weighted average, sample sizes as weights). Hence, the current research focus is in the case of VI people biased in favor of the younger part of the population.

User research conducted in a specialized residential care facility for visually impaired older adults [4] showed that clients are also often challenged with other age-related health issues. Frequently, those people are challenged with cognitive issues (mainly dementia), or mobility issues. The facility provides individualized care based on personal psychobiographical modeling of individuals [5], [6].

In our previous research, we focused on the development of solutions to support orientation in space tailored to needs and preferences of visually impaired older adults [4]. The evaluation indicated that adaptation of user interfaces and interaction method based on individual needs and preferences could improve usability of particular solutions as well as maximize their acceptance among the target user audience.

In this paper, we present a user modeling approach tailored primarily for the user group of visually impaired older adults living in residential care facilities. The user model can represent individual aspects related to personal biography as well as aspects important for adaptation of user interfaces and interaction methods.

\section{RELATED WORK}

In this section, we discuss various technical approaches to represent personal attributes related to interaction with other human beings as well as with technologies - user models. Furthermore, we describe specific approaches for geriatric and gerontopsychiatric care. Finally, we list technological approaches where user model is used for adaption of user interfaces and interaction methods and for providing personalized care.

The original user modelling approaches emerged from the medicine and rehabilitation engineering. The World Health Organization (WHO) comes up with models, which are based typically on measurement and quantification of human performance, which is examined in rehabilitation engineering. The WHO defined ICD (International Statistical Classification of Diseases and Related Health Problems) [7] and ICF (International Classification of Functioning, Disability and Health) [8].

Ability-based design method proposed by Wobbrock et al. [9] suggests leveraging specific individual abilities rather than focusing on disabilities. The use of user-specific abilities and capability of interactive systems to adapt accordingly could make the interaction more efficient, natural, and broaden the audience of users that can successfully use a particular technology.

Peißner et al. [10] come with the idea of individual patterns for accessible and adaptive user interfaces, which are built on information about the user, the context and the devices, 
which is gathered through system interaction and sensors. All information about the user is stored in the User profile, which variables include his abilities, disabilities, preferences and his current environment. All the values of the user profile are numeric between zero and four. The context manager continuously updates the user profile. The user profile mixes different variables, and some of them are not so typical for user models (e.g., ambient light and ambient noise).

Heckmann et al. [11] introduce the general user model ontology (GUMO) for the uniform interpretation of distributed user models in intelligent semantic web enriched environments. GUMO uses UserML (User Model Markup Language) [12], which is Resource Description Framework (RDF)-based user model exchange language.

Kikiras et al. [13] present a user model for navigation systems, which is represented through a Semantic Web ontology (User Navigation Ontology - UNO). For that purpose, they use the Web Ontology Language (OWL) for describing the user classes and their properties. During ontology development, they extended some of the concepts specified in the GUMO ontology [11]. They also adopted the International Classification of Functioning, Disability and Health (ICF) [8] of World Health Organization (WHO) for representing certain functioning and disability issues of an individual. Their model comes up with so-called "User profile", which captures user's demographics, mental-cognitive characteristics, sensory abilities, motor capabilities, navigational preferences and interface preferences.

The Böhm's model [5], [6] is an internationally recognized nursing model which is currently used at most in Germanspeaking countries in the field of geriatric and gerontopsychiatric care. The model is aimed to support the self-care of old and confused people and it is also focused on how to retain or restore the self-care ability for as long as possible by the principle of recovery of the senior's interest by reviving his psyche. Daily life and normality for patients is one of the core issues of Böhm's nursing model. The theory of Böhm is based on understanding of patients, their biography and coping strategies. The biographic assessment and knowledge of coping strategies is crucial for the psychobiographic nursing concept. Patients should stay as long as possible independent and self-reliant and should keep their social competence. Böhm comes up with the theory that learning copings takes place in the first 25 years of life, and in regression, one returns to their own lower copings.

Hoey et al. [14] present a summary of customizable and adaptive technologies for assistance for persons with cognitive disabilities. Authors describe decision-theoretic model based on Partially Observable Markov Decision Process (POMDP) that can be applied to various activities. The system allows user customization, system adaptivity to user and general purpose sensing abilities. Authors state that the concept of inclusive design works only for large and uniform segments of population, but fails for individuals with diverse and changing needs like older adults with Alzheimer's disease. A system itself must adapt to people over time as they change.
Internet of Things sensor network as source of heterogeneous contextual information is mentioned in [15]. The contextual information (e.g. daily activities) is integrated into personalized care management processes to support automatic and better decision making in an effective and user-friendly manner. There is set of sensors that recognize personal activities, and the system can provide guidelines how to proceed when one experience an issue when carrying out a particular task. These context-aware services can help older adults to stay at their homes safelly.

There are several approaches for user modeling, however, only few leverage specific user abilities (what user can do and can be used for interaction) rather than representing disabilities (what user cannot do and must be compensated by assistive technologies). User group of visually impaired older adults requires a specialized approach for both care provided and interaction with technologies. User research described in section III leverages these specifics. The available user modeling approaches cannot represent the necessary user attributes to a sufficient extent. Also, there is a lack of technological support for psychobiographical modeling of individuals and corresponding care model.

\section{USER STUDY}

To learn more information about application of psychobiographical care model in practice, the user research was conducted in the institution for visually impaired people (104 employees and 125 clients in 2019).

\section{A. Participants}

User research was conducted with three participants $(\mathrm{P} 1-$ P3), all women, average age $44.33(S D=15.95, M I N=26$, $M A X=55) . P 1$ and $P 2$ work as activation services workers and $P 3$ works as direct care worker (social worker). None of the participants worked in a similar type of facility before working in the residential care home we cooperate with. Mean duration of current job title was 7.83 years $(S D=7.18)$.

\section{B. Procedure}

The user research has the form of a qualitative semistructured interviews with employees of the institution for visually impaired people. The topics were focused on the activities of activation services workers and direct care workers, the usage of the psychobiographical care method in practice, and the usage of technologies in the institution. One interview lasted approximately 35 minutes (short briefing, 30 minutes interview and short debriefing).

\section{Results}

Job description. The main task of the activation services worker's job is to give clients the opportunity of selfrealization even in older age. Activation services workers prepare for clients various types of activities, both individual and group. These include, for example, cooking, singing, memory training and many more. The activities are tailored to individual clients according to their preferences and habits 
from their previous life and are often related, for example, to the client's profession. All activities are voluntary, and it is up to clients whether they want to do any of them. Activation services workers cooperate with health professionals and direct care workers, and it is very important to create one compact team of workers.

Direct care workers are responsible for the social aspects of care. They help clients with a wide range of activities, such as personal hygiene, dressing, moving, and eating. An important part of their work is also communication with clients and supporting them in their favorite activities. Every direct care worker is in charge of three clients as a so-called "key worker". He/she should know the most about these clients, map their needs and preferences and share this information with the entire team.

Daily programme. The residential care home daily programme has three reference points - breakfast, lunch, and dinner. Between breakfast and lunch, and then between lunch and dinner, there are group, as well as individual, activities that are provided by activation services workers and direct care workers. For clients, the program is voluntary, and it depends only on them what activities they choose, each client has different interests and preferences. Within the individual activities, workers chat with clients, read, sing, go out, etc. There are plenty of group activities in the residential care home - rehabilitation exercises, workshops, singing, crosswords solving, memory training, art therapy, canistherapy, music therapy and more. Group activities take place regularly, usually once a week. Cultural events, such as concerts or theatre performances, are held twice a week at the Great Hall, and these events are very popular. There are also held, approximately four to five times a year, excursions outside of the residential care home, for example, trips to castles and chateaus, trips to theatres and a trip by steamboat.

Technology use by clients. The use of computers and similar technologies is limited in the residential care home due to various limitations of clients. Most clients also have a barrier between them and modern technologies. "People, clients, who come here can't work with modern technologies. Maybe they have never seen a computer during their lifetime." (P2)

In the residential care home, there is a device called a reading magnifier, which is used by some clients. In some departments, some gramophones are used to play old records and also to induce the atmosphere of the times when gramophones were commonly found in households. In addition, they use voice-output clocks to inform clients of the current date, time, weather, and so on. Unfortunately, for most clients, these clocks are too complicated. "The voice clocks we use here are too complicated. For most clients, clocks report too much information, which can lead to a big cognitive burden on clients." (P3)

Biography and Böhm's psychobiographic method.

In the residential care home, Böhm's psychobiographical model of care is applied, specifically its modification, which is adapted to work in the institution. "We use the Böhms method adapted for our needs. " (P3) The client's biography is a key element of the psychobiographical model. "Biography is such a client's book of life. " (P2)

The process of collecting information into biography and its processing begins in the period before the client comes to the residential care home. In this period, the client and his family may be asked to provide some information. "Even before the client arrives, the client and his/her family are asked to think about providing information and photos of the client and to write a client's life story at their discretion." $(P 2)$ Unfortunately, cooperation with family and clients is sometimes difficult. "Sometimes collaboration with client's family is complicated because they are reluctant to provide sensitive information about a close person. " $(P 2)$ However, it is essential that neither the client nor his/her family is pushed into anything and that the provision of information must be based on a free decision.

After the client's entry to the facility, the "key worker" assigned to the client has to start the processing of biography. "We then communicate with the client and start processing the mapping, where we write down the information that client provides to us. Based on it, we create the client's life story, which is written into a biographical book with attached photographs. “ $(P 2)$ Workers must establish a relationship with the client to get as much information about the client as possible. "The amount of information that clients are willing to convey is different, someone is willing to tell everything, someone almost nothing. It is up to us to establish a relationship with the client to tell us something, but at the same time, we must understand that client have told the information to us and may not want the information to appear somewhere. I had a client who directly wished for some information not to appear anywhere and did not even want to publish information under her name, so she invented a fictitious one. " (P3)

Biography is in paper form. It has a fixed structure according to which workers must proceed during its creation process.

In the biography, there is a client's life story with photographic documentation. The life story contains information about the client's childhood, youth, adulthood, old age, and this information is then used to work with the client. There is a need to find out what the clients have gone through in their lives, about their families, their interests, their education, their occupations and then specific patterns of behaviour can be derived from this knowledge. "Biography should tell us about client's behaviour patterns, in which environment he grew up, and we must realise that man in old age is hardly going to change his behaviour. " $(P 3)$ It is also necessary to put the information about the client's life in the historical context. Many clients have experienced the First Czechoslovak Republic, the Nazi occupation, the putsch in 1948, the totalitarian regime, the Soviet invasion in 1968, and of course all these events influence man. "We should have a basic, maybe even advanced, knowledge of history to know what it does to a man when he experiences during his active life such turbulence as in the 20th century in Europe, especially in Czechoslovakia." (P3) It is also necessary to learn negative information about 
the client to avoid possible misunderstandings and unpleasant situations. "It is also good to know topics that client doesn't want to talk about. Some clients have experienced war or totalitarianism. Some of them don't mind talking about it, but some clients don't want to hear German or Russian. So it is necessary to know negative information, experiences, etc. " (P2)

Also, the biography includes a client's tree of life (lineage), where information about his relatives, friends, etc. can be found. "In deeper dementia, clients become more likely to think that we, caregivers, are their friends when they were ten years old and they address us with the names of their friends. And when we know that he/she is addressing us on behalf of a friend, we know he/she has such a relationship with us. "(P2)

Furthermore, so-called "activities of daily living" are part of the biography. These are divided according to the tasks of the ordinary day. They describe what is typical for the client, what he was used to and how to transfer this fact to residential care home reality as faithfully as possible so that the client feels at home. The biography also affects activities such as client dressing up, which can be taken as an example of such a task of daily living. "We had a client who belonged to the almost highest social class in the First Czechoslovak Republic. She was very fond of wearing luxury costumes with pearls. Thus, in dressing, it would be written in the activities of daily living, that she was used to the luxury, that she still keeps these habits and that even when she is infirm, we will not give her sweatpants even if it is easier for us to provide her with care. " (P3)

If a client with dementia enters the facility, it is necessary to collect the information as soon as possible. "When a new client with dementia comes here, we need to find out as much information as soon as possible. There is a need for cooperation with the client's family. It may happen that the client enters the residential care home and in two months period, he will not be able to provide us with further information. Then, work based on the biography is complicated. “ (P2)

Biography is constantly maintained and updated with new information. "Biography is handled by everyone except medical staff. Maintenance of biography is the work of the whole team." (P2) Access to biography, which is located in the nurses' room in locked safes, is available to all department workers and can be provided to the client or his/her family upon the client's request. "Biography contains sensitive personal information, often even intimate, so trainees or anyone else who does not work in the department does not have access to it." (P2) Employees have regular weekly meetings where they discuss individual clients. "We always have a meeting on Wednesday, where clients are discussed according to Böhm's method. We all, including health professionals and a psychologist, discuss the client's score (differential diagnosis), the activities of daily living and so on. The key worker then selects all the relevant information he needs for further working with the client. " (P2)
The biography is fundamentally reflected in the work of caregivers. "Activation and communication with the client are tailored to the biography." (P2) "For example, the client was a seamstress all her life, so she works with a fabric that reminds her of a long period of her life. " (P1)

Based on a biography, caregivers try to bring clients closer to the environment they have been familiar with during their active life. For example, listening to music and recordings from that period can help. "The music of the 1950s is playing right next door - Chladil, Simonová and other singers. We play recordings from Semafor every day, so these days we get slowly to 1960s. " (P2) Then, for example, reading books about the time, when clients actively lived, about places, which clients could visit and about the activities they could perform can also help to induce well-being. "We read autobiographies of actors, musicians, and writers. We also read about countries and places where clients often travelled in the 1970s - Hungary, Poland, East Germany, we read for example, about Rügen and Lake Balaton. We also read about camping or pigslaughtering. " $(P 2)$ The aim is to retrospectively return clients to a time when they were actively living their lives. "We are going back to the age when clients were about 25 years old." (P2)

For less oriented clients, workers read fairy tales, various stories and poems. "We read mainly the fairy tales of Božena Němcová, Karolína Světlá and other authors. We read especially the shorter ones, such as The Red Riding Hood. We also read some poems that are clients familiar to." (P2)

The activities of clients and activities of workers with clients are recorded in the system. However, clients have the freedom of movement around the residential care home, and so, for example, a visit from a client's family is not recorded. "Clients have freedom of movement, but most of them report such activities. If the client leaves the home, we write down the fact that the client left and also the expected return." (P2)

"The biography must be handled carefully, and workers must maintain a professional approach without an evaluation approach, even though some client's habits may, for example, seem weird to them." (P3) "The problem is with the increasing difference between clients and workers. There are two completely different worlds, the world of young workers on one side and on the other side the world of clients who are still ageing. Therefore, the worker must understand the client, which is also very difficult and some misunderstandings may arise. " (P3)

Technology use by workers. A barcode readers system for recording client's activities, which is used in the residential care home, helps employees. Each activity is assigned a unique barcode that is scanned with the reader and all records are downloaded to the system at the end of every day.

All participants can imagine the electronic version of the biography, but the current form is more acceptable and enjoy- 
able for most of them (P2 and P3). "It's nice to have it in the form of the book, it's more natural and easier to pass on to someone. " (P2)

The user research with employees of the residential care home indicated that personal psychobiography is frequently used for purposes of providing care. The collection of data begins even before a client arrives at the institution. It contains information about client life in the historical context, their specific patterns of behavior, or activities such as client dressing customs. Furthermore, the biography contains so-called "activities of daily living" that describe what is typical for the client and what he was used to. The psychobiographical sheet, which includes all the relevant information about the client's biography and nowadays is in the paper form, is shared among personal, and there is limited access for others. Workers also routinely record activities performed with clients into the system using a barcode scanner. The research with employees and our previous research with clients described in [4] showed, that clients have a barrier in using modern technologies. However, our previous research showed that technological products that are well-adapted to needs, preferences and abilities of them could help clients with daily activities like orientation [?] or leisure [16].

\section{Proposed Solution}

The whole solution consists of the user model, user interface for its maintenance and appropriate API, which will provide all the relevant information from the user model to user interface and client's devices. The architectural overview is depicted in Figure 1. The user model and user interface are described in detail in following sections. The solution also takes into account the connection to an existing system from which records of client activities could be obtained (see Section III). Various devices will be connected to the solution. There are two groups of users, whom the solution will serve primary group, represented by the workers (caregivers) of the residential care home and secondary group, represented by the clients, who will use the user model indirectly through their devices and provided care.

\section{A. Proposed User Model}

Based on the analysis and requirements of other projects, there can be deduced information categories - user model components:

1) Demographics: This category contains basic information about client including the client's name, surname, birth date, birthplace, address, gender, education, department, room number, key worker, contact persons and photographs of the client. The user model also allows to model and maintain the relationship between client and workers. The relationship also captures the rights of workers (view/edit various information categories).

2) Biography: The client's biography is divided into four sub-categories. The first one is the biographical sheet, which represents the life story of the client. Then, there are the

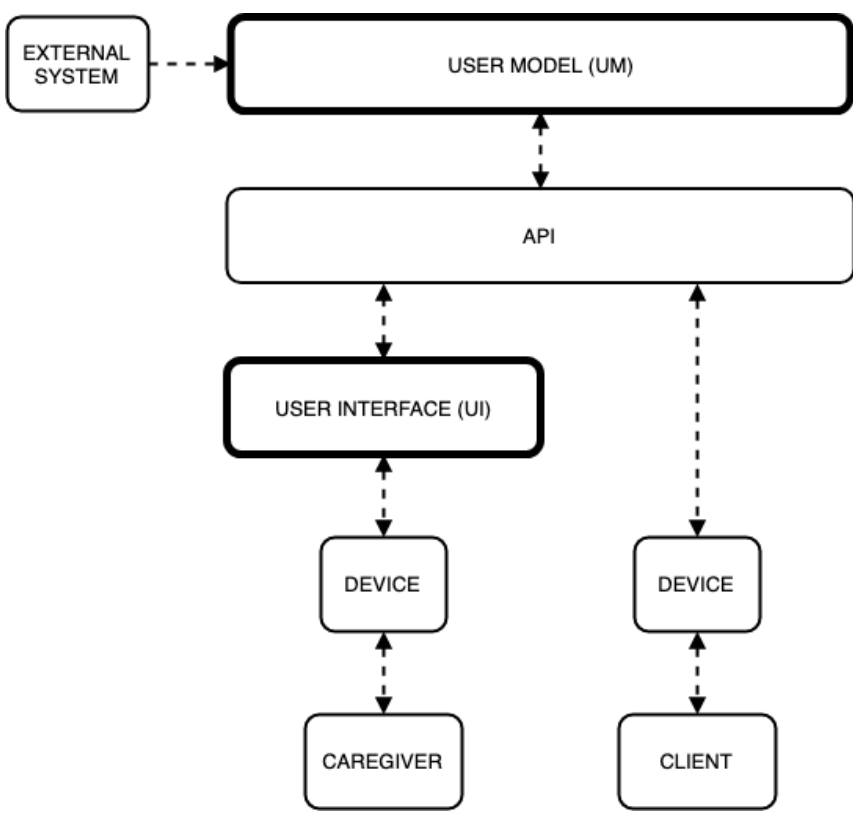

Fig. 1. Proposed solution - High level view.

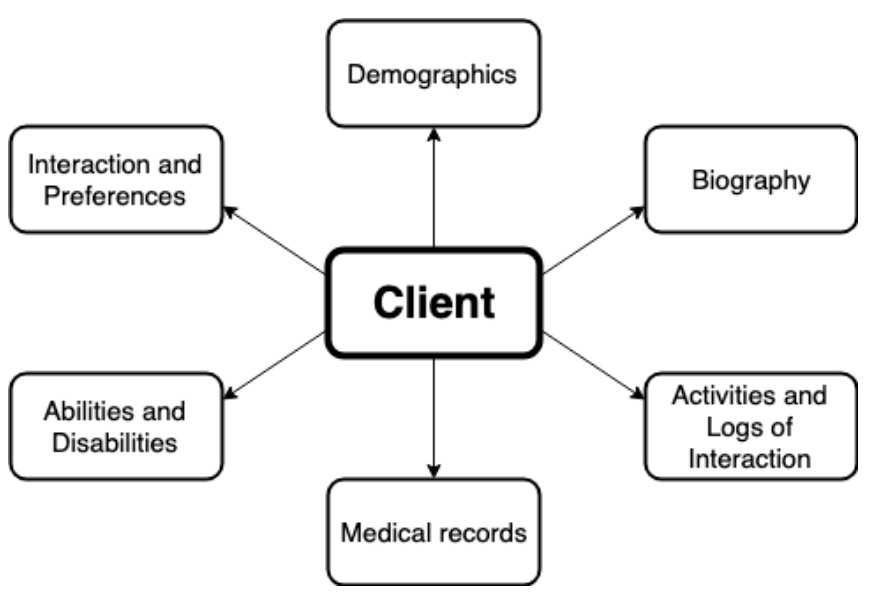

Fig. 2. User model components.

activities of daily living, which are represented by seventeen different tasks of the ordinary day (see Section III). The next category is the lineage which captures the family tree of the client. And finally the form of care, which is composed of three different types of care (activation, reactivation and stimulation) and the differential diagnostics score (eight areas of older adult behavior - psychomotor, orientation, emotions, memory, contact ability, formal thinking, will and content thinking).

3) Activities: This category contains records of the client's activities. According to the conducted user research (see Section III), the employees use a barcode readers system for recording client's activities. Each activity is assigned a unique barcode that is scanned with the reader and all records are downloaded to the external system at the end of every day. There are also logged the activity of the clients during interaction with devices, which will bring the possibility of 
device adaptation.

4) Medical records: To this category belongs medical records of the client including the client's height and weight, diseases, injuries, allergies, diets and prescribed medication.

5) Abilities and Disabilities: The abilities and disabilities of the client are divided into three sub-categories - sensory, motor and cognitive.

The sensory category contains information about sensory abilities and disabilities of the client. Information is divided into sight, hearing and touch, additionally for each eye, ear and hand separately.

The motor category captures mobility information, such as motor impairment but also, for example, the ability to stand alone or ability to move independently.

The cognitive category contains information about cognitive abilities and limitations of the client, for example, the client's knowledge of his room or level of independence.

Information about impairment captures the severity, origin, progression and other characteristics of the impairment. There are also captures of the abilities of the client, for example, the ability to perceive object shapes or level of client's tactile abilities. If the attribute is expressed in scale, a range of 0 to 6 is used. For example for the visual impairment, the ICD $9 D 90$ classification [17] is used.

6) Interaction and Preferences: This category contains information about interaction with client and preferences of the client (for example client's dominant hand or dominant modality), including the client's attended activities, hobbies, favourite places, interests in services and client's equipment.

The categories of the user model are depicted in Figure 2.

\section{B. Administration UI Design}

For the purpose of the creation of the prototypes, two different development tools were used. Each prototype has been implemented to support the interaction needed to accomplish the tasks performed during prototype evaluation.

1) Low-fidelity prototype: A very first prototype (lowfidelity prototype) of the user interface was created in the form of a paper prototype and was implemented using tool Balsamiq Mockups [18]. All application screens and all necessary interaction elements were implemented. The prototype was then evaluated in paper form (see Section V-A).

The structure of the proposed user interface reflects the user model structure (see Section IV-A) and user interface requirements, collected in time before the creation of the low-fidelity prototype. The goal was to create a prototype that is easy to maintain and minimalist, clean and intelligible. This prototype was evaluated in the usability study with three representatives of the target group. The user interface consists of five main screens, which further branches into sub-screens. The complete structure of screens can be seen in Figure 3.

Login page. The Login page is the first screen the user encounters when interacting with the application. The screen allows the user to enter username and password to log into the system.
Home page. The Home page acts as the main application signpost. It allows the user to go to the client search screen or to add a client screen. At the top of the screen, there is a panel that informs the user about the current location, allows the user to view notifications, displays the user's name and surname, and allows the user to log out of the system. This panel then appears on all other screens.

List of clients page. The List of clients page displays the list of clients and allows the user to apply filtration of records.

Client detail page. The Client detail page consists of seven sub-pages:

\section{- Basic information.}

The Basic information screen displays basic information about client. As an example of the low-fidelity prototype, screen can be seen in Figure 4.

\section{- Biography}

The Biography consists of four sub-pages:

- Biography - Biographical sheet.

The Biography - Biographical sheet screen displays biographical sheet text and attached photographs. The user is able to edit text and maintain attached photographs.

- Biography - Activities of daily living.

The Biography - Activities of daily living screen displays table with seventeen different activities of daily living. The user can edit each table row (activity) separately.

- Biography - Lineage.

The Biography - Lineage screen displays the family tree of the client. The user can maintain the lineage.

- Biography - Differential diagnostics sheet.

The Biography - Differential diagnostics sheet screen displays table with differential diagnostics score of the client and corresponding form of care.

\section{- Activities.}

The Activities screen displays activities of client. The screen is only used to show activities, the data is located in an external system (see Section IV). The user can filter activity records.

\section{- Medical records.}

The Medical records screen shows health information. It shows the client's height and weight and further diseases, injuries, allergies, diets and prescribed medication. The user can maintain all this information.

\section{- Abilities and disabilities}

The Abilities and disabilities consists of three sub-pages:

- Sensory.

The Abilities and disabilities - Sensory screen shows information about the client's sensory abilities and disabilities, such as severity, origin, progression and other characteristics of visual, hearing and tactile impairment and also corresponding abilities of the client, for example, ability to perceive object shapes.

- Motor.

The Abilities and disabilities - Motor screen shows 
information about the client's motor capabilities, such as severity, origin, progression and other characteristics of motor impairment and also corresponding abilities, for example, ability to stand alone.

\section{- Cognitive.}

The Abilities and disabilities - Cognitive screen shows information about the client's cognitive capabilities, such as the ability to read Braille, knowledge of the room, knowledge of residential care home interior and other abilities.

\section{- Interaction and preferences.}

The Interaction and preferences screen displays information relevant to client interaction and client's preferences, such as dominant hand, primary modality etc. On the screen, there are also tables for records of client's attended activities, hobbies, equipment which client use, favourite places and interests in services. The user can maintain all this information, including adding and removing records in tables.

\section{- History.}

The History screen displays a history of client information changes. The user can filter records of changes.

Add client page. The Add client page allows the user to add a client into the system. Some fields are marked with an asterisk, indicating that they are mandatory. The user has the option to save the new record (and get on the Client detail page screen) or to cancel the process of the creation and return to Home page.

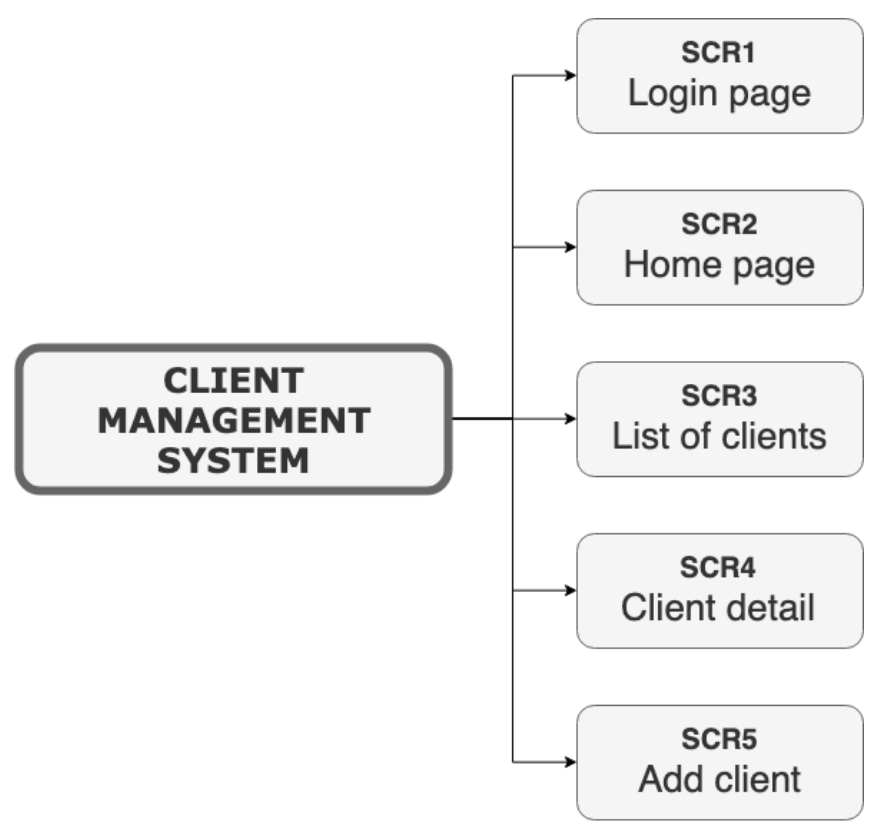

Fig. 3. Low-fidelity prototype - overview.

2) High-fidelity prototype: After the low-fidelity prototype evaluation, the high-fidelity prototype was implemented. The tool Axure [19], which enables the creation of more advanced prototypes, was used for this purpose. The prototype was then

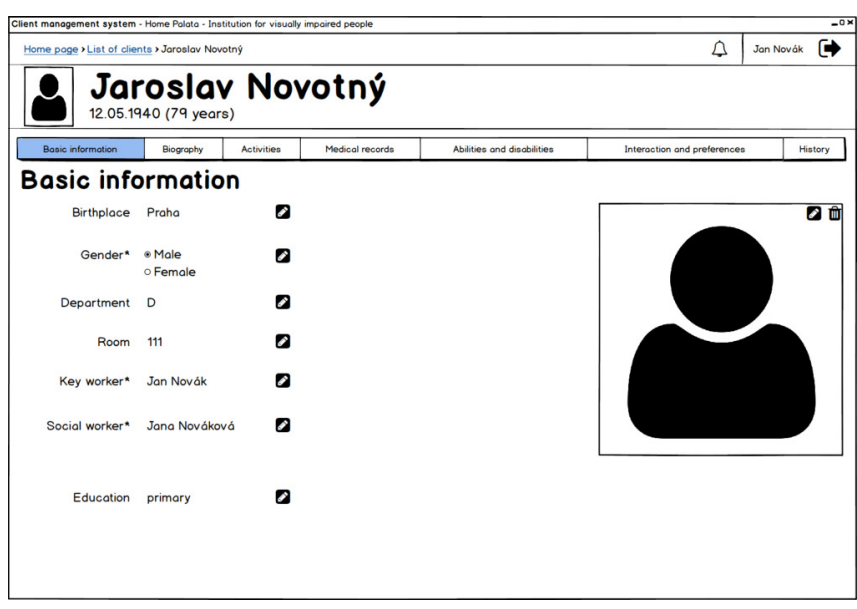

Fig. 4. Low-fidelity prototype - SCR4a - Client detail - Basic information.

evaluated in the form of interactive computer application (see Section V-B).

The concept of the user interface remains the same as the low-fidelity prototype. There are used screens specified in the low-fidelity prototype but the visual appearance of the user interface is improved to get closer to the final product. The experience is also enhanced by the implementation of simple animations or effects, for example, animation of expanding notification detail or buttons hover effect. There are also changes based on the requirements, comments and suggestions, gained during the low-fidelity prototype evaluation as well as changes reflecting the findings of the evaluation of the low-fidelity prototype. The example high-fidelity screen prototype can be seen in Figure 5.

As described above, there are some changes based on the requirements, comments and suggestions, gained during the low-fidelity prototype evaluation and also changes reflecting findings of the evaluation of the low-fidelity prototype, see section V-A. The main changes are listed below:

\section{Naming of screen Differential diagnostics sheet}

The naming of the screen Differential diagnostics sheet has been changed to Form of care, which should eliminate the problems that occurred during low-fidelity prototype evaluation.

\section{Highlight of notifications}

The notification is highlighted by red color. This change should fix the problem of feeble notification, that was faced by participants during low-fidelity prototype evaluation.

\section{Unsaved data alert}

The notification about the unsaved data has been added to the prototype. This should eliminate the situation, when users omitted the final confirmation of data change.

\section{Extended attribute values options}

Text description is added for "other" attribute value option. 
Also, an undefined value of attributes, which values are selected through radio buttons is possible.

\section{Client information print}

There has been added a possibility to print out the information about the client.

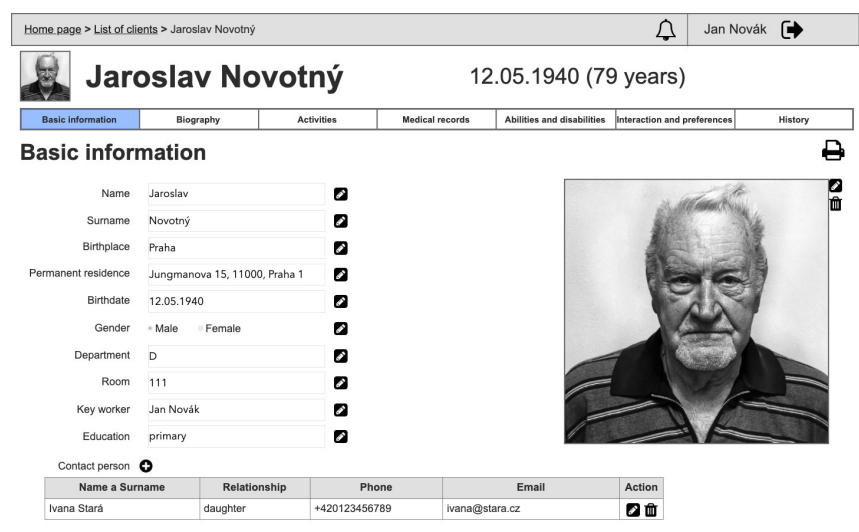

Fig. 5. High-fidelity prototype - SCR4a - Client detail - Basic information.

\section{Evaluation}

We evaluated two generations of prototypes of the administration UI with employees of the residential care home. The relatively low number of participants was determined by complicated methods to reach such a specific user group and correspondingly high costs per participant. More information about determining sample size for hard-to-reach user audience can be found in [20], [21].

\section{A. Administration UI Design Low-Fi prototype}

Participants. Low-fidelity prototype evaluation was conducted with three participants (P1-P3), all women, average age $33.33(S D=11.09, M I N=25, M A X=49)$. All participants work as activation services workers. One of the participants $(P 2)$ was previously interviewed in the user research. None of the participants have participated in usability study before. Mean duration of current job title was 1.33 years $(S D=0.47)$.

Procedure. The usability study was conducted with employees of the institution for visually impaired people. The test session was under non-laboratory conditions, and it took maximum of an hour. Paper low-fidelity prototype and The Wizard of $\mathrm{Oz}$ technique were used. The participants were recorded on a camera in order to log the testing session afterward. Firstly, the participants were informed about the process of the testing session. After the briefing, they filled in a pre-test questionnaire. Then, the system (application) was introduced to them briefly, and they were informed about the purpose of the system. Participants were encouraged to comment aloud their activities. After that, participants were asked to complete a list of three complex tasks focused on intended typical interaction with the user interface:

- logging in and out

- searching for a client

- viewing information

- editing information

- adding client

After completing all tasks, participants filled in the post-test questionnaire. Finally, participants were asked to share their opinion on the application and on the testing session.

Results and conclusion. During the usability study of the low-fidelity prototype, the basic functionality of the user interface was verified.

The usability study also revealed three problems of the proposed design, which must be fixed in the next stages of the design process. The biggest problem faced by all participants was the incomprehensible and misleading term "Differential diagnostics sheet", which was used based on literature analysis. It turned out that none of the participants could imagine anything under this term. This problem can be fixed by renaming the item to "Form of care", which was also suggested by all the participants. Another, quite fundamental problem was feeble notification, which was represented by a tiny black circle. This problem was faced by two participants. The solution to this problem is to make notification more visible. And the last problem, also faced by two participants, was the problem of leaving out the final confirmation of the information editing. The solution is to notify the user, if he/she does not confirm editing and he/she intends to move to another screen. Highlighting the buttons could also help to improve interaction.

The usability study also provided valuable feedback from the participants, and the knowledge gained during testing and post-test interviews can be useful to improve the design of the user interface. Post-test interviews also brought a requirement for new functionality - printing of client information.

The positive information is that all the participants quickly became familiar with the application, even searching for information (attributes) has become easier after a while. All the participants also emphasise the clarity of the proposed design.

\section{B. Administration UI Design High-Fi prototype}

Participants. High-fidelity prototype evaluation was conducted with six participants (P1-P6), all women, average age $43.5(S D=14, M I N=26, M A X=64)$. Three participants work as activation service workers $(P 1, P 2, P 3)$, two participants as direct care workers $(P 4, P 6)$ and one participant (P5) works as speech and occupational therapists. Two of the participants $(P 2, P 3)$ have participated in previous low-fidelity prototype evaluation. Mean duration of current job title was 6.87 years $(S D=4.7)$.

Procedure. As same as low-fidelity prototype evaluation, the usability study of the high-fidelity prototype was conducted with employees of the institution for visually impaired people. The test session was under non-laboratory conditions, and it took a maximum of 45 minutes. Participants were using 
a computer (MacBook Pro, screen size 13 inches, Google Chrome), and remote computer mouse. The screen of the computer was recorded with sound captured by the internal laptop microphone to log the testing session afterwards. Firstly, the participants were informed about the process of the testing session and after the briefing, they filled in a pre-test questionnaire (the questionnaire contained the same questions as in the low-fidelity prototype evaluation). Then everything went on the same way as evaluation of the low-fidelity prototype, the system (application) was introduced to participants briefly, they were informed about the purpose of the system and they were encouraged to comment aloud their activities. After that, participants were asked to complete a list of three complex tasks focused on intended typical interaction with the user interface. After completing all tasks, participants filled in the post-test questionnaire (the questionnaire contained the same questions as in the low-fidelity prototype evaluation). Finally, participants were asked to share their opinion on the application and the testing session.

Results and conclusion. During the usability study of the high-fidelity prototype, the more advanced functionality, compared to low-fidelity evaluation, of the user interface was verified.

The usability study revealed three problems of the proposed design, which may be fixed before the final product will be released. The biggest problem faced by all participants was a difficulty of searching for individual attributes. The problem is caused by a large number of attributes and also the ambiguity of distribution into the individual categories. The problem can be fixed by adding a search box, which can be used for the individual attributes search. Another problem is the unclear difference between screens Activities and Activities of daily living. Five participants out of six have faced this problem. During the first walkthrough through the application, screens naming may be confusing and also, Activities of daily living (SCR4b2) screen is hidden by default as a Biography sub-page. The solution is to rename the screen Activities to improve user orientation in the application, for example, the screen could be renamed to "Activities of the client". And the last problem, which was faced by three participants, were small and feeble radio buttons and checkboxes. The participants complained about the size of the radio buttons and checkboxes and these interaction elements were also very feeble in their point of view. This problem can be fixed by increasing the size of active elements of the interaction.

The knowledge gained during testing and post-test interviews can be very useful to improve the design before releasing the final product. Post-test interviews also brought a requirement for new attribute - information when the client joined the residential care home.

Despite all the problems faced by the participants during the application evaluation, very positive feedback on the application's clarity was obtained.

\section{DISCUSSION}

The evaluation of both generations of the prototypes indicated that the user interface is generally clear for employees of the residential care home. Although some employees in the user study indicated that they like the paper form of the biographical sheet, the electronic form can bring further benefits. It would be possible to track the progress of clients disease by analyzing the biographical sheet and activities performed with the client. Also, the electronic form will bring better privacy security by authorized access and by tracking the access to the biographical sheet.

The validity of the user study and evaluation is limited due to relatively low number of participants and the fact the research has been conducted in a single residential care institution. However, there is a strong evidence that older adults, especially those with cognitive issues like Alzheimer's disease require personalized care. Also, interaction with technologies needs to be adapted to their needs, preferences and changing abilities. This fact is also supported by literature, e.g., [14].

Mutual connection of the personal psychobiography [5], [6] and user profile in one user model could bring various benefits for interaction with technologies. It would be possible to adjust a user interface according to the needs and preferences of a particular client. Properties like rate of speech, information complexity, the volume can be automatically considered. Also, a significant part of the clients has some remaining residual sight that can be used in interactive scenarios. Knowing particular client abilities can improve interaction significantly. For instance, some clients have central vision quite intact, others have peripheral vision only while some can only sense high contrast patterns. Also, the personal psychobiography can be used to make the interaction more natural and personal. Data in the user model can be used for client identification utilizing biometry. Upon this, it is possible to address the client by her/his name in an appropriate form. Also, technological applications like interactive indoor orientation system can provide personalized information like instructions on how to navigate to one's own room

\section{CONCLUSION}

For the specific user group of older adults with vision impairments, we proposed a solution that consists of the user model structure, corresponding administration user interface and appropriate API. The user model design connects aspects of personal psychobiography that is already successfully applied in the gerontological care and aspects that are important for interaction with technologies.

It is the subject of the future work to fill the user model with data of real clients of the residential care home. Then, the model will be evaluated for the purposes of providing personalized daily care. Furthermore, technological applications will use the model for adaptation of user interfaces and interaction. It would be possible to adapt properties like information complexity, speech rate, or volume. Also, it will be possible to provide more personal communication by addressing the client by her or his name in an appropriate way. 
Selin and Rossi [22] propose a method to design safer buildings based on information models. They propose to use Building Information Modelling (BIM) for simulation of various situations individuals can deal with in an interior, including evacuation. They use a gaming engine and artificial intelligence to simulate individual with different capabilities. It is the subject of the future work to incorporate modeling of the indoor environment and the ability to plan routes based on needs, preferences, and capabilities of individual users.

\section{ACKNOWLEDGMENT}

This research has been supported by the TACR research program TE01020415 and the project RCI (reg. no. CZ.02.1.01/0.0/0.0/16_019/0000765) supported by EU and by the project Navigation of handicapped people funded by grant no. SGS19/178/OHK3/3T/13 (FIS 13139/161/1611937C000).

\section{REFERENCES}

[1] R. R. Bourne, S. R. Flaxman, T. Braithwaite, M. V. Cicinelli, A. Das, J. B. Jonas, J. Keeffe, J. H. Kempen, J. Leasher, H. Limburg et al., "Magnitude, temporal trends, and projections of the global prevalence of blindness and distance and near vision impairment: a systematic review and meta-analysis," The Lancet Global Health, vol. 5, no. 9, pp. e888-e897, 2017. doi: 10.1016/S2214-109X(17)30293-0. [Online]. Available: https://doi.org/10.1016/S2214-109X(17)30293-0

[2] World Health Organization, World report on ageing and health. World Health Organization, 2015.

[3] Český statistický úřad. (2014) Výběrové šetření zdravotně postižených osob - 2013. [Online]. Available: https://www.czso.cz/csu/czso/ vyberove-setreni-zdravotne-postizenych-osob-2013-qacmwuvwsb

[4] M. Macik, I. Maly, J. Balata, and Z. Mikovec, "How can ict help the visually impaired older adults in residential care institutions: The everyday needs survey," in 2017 8th IEEE International Conference on Cognitive Infocommunications (CogInfoCom). IEEE, 2017. doi: 10.1109/CogInfoCom.2017.8268234 pp. $000157-000164$. [Online] Available: http://dx.doi.org/10.1109/CogInfoCom.2017.8268234

[5] E. Böhm and P. Sochová, Psychobiografický model péče podle Böhma. Mladá fronta, 2015.

[6] E. Procházková, Práce s biografií a plány péče. Mladá fronta, 2014.

[7] World Health Organization, International statistical classification of diseases and related health problems. World Health Organization, 2004, vol. 1.

[8] World Health Organization and others, International classification of functioning, disability and health: ICF. Geneva: World Health Organization, 2001.

[9] J. O. Wobbrock, K. Z. Gajos, S. K. Kane, and G. C. Vanderheiden, "Ability-based design," Communications of the ACM, vol. 61, no. 6, pp. 62-71, 2018. doi: 10.1145/3148051. [Online]. Available: http://dx.doi.org/10.1145/3148051
[10] M. Peißner, D. Janssen, and T. Sellner, "Myui individualization patterns for accessible and adaptive user interfaces," in The First International Conference on Smart Systems, Devices and Technologies, 2012, pp. 2520.

[11] D. Heckmann, T. Schwartz, B. Brandherm, M. Schmitz, and M. von Wilamowitz-Moellendorff, "Gumo-the general user model ontology," in International Conference on User Modeling. Springer, 2005, pp. 428 432.

[12] D. Heckmann, T. Schwartz, B. Brandherm, and A. Kröner, "Decentralized user modeling with userml and gumo," in Decentralized, Agent Based and Social Approaches to User Modeling, Workshop DASUM-05 at 9th International Conference on User Modelling, UM2005, 2005, pp. 61-66.

[13] P. Kikiras, V. Tsetsos, V. Papataxiarhis, T. Katsikas, and S. Hadjiefthymiades, "User modeling for pedestrian navigation services," in Advances in ubiquitous user modelling. Springer, 2009, pp. 111-133.

[14] J. Hoey, C. Boutilier, P. Poupart, P. Olivier, A. Monk, and A. Mihailidis, "People, sensors, decisions: Customizable and adaptive technologies for assistance in healthcare," ACM Transactions on Interactive Intelligent Systems (TiiS), vol. 2, no. 4, p. 20, 2012. doi: 10.1145/2395123.2395125. [Online]. Available: http://dx.doi.org/10.1145/2395123.2395125

[15] L. Yao, B. Benatallah, X. Wang, N. K. Tran, and Q. Lu, "Context as a service: realizing internet of things-aware processes for the independent living of the elderly," in International Conference on Service-Oriented Computing. S Springer, 2016. doi: 10.1007/978-3-319-46295-0_54 pp. 763-779.

[16] B. Endrstova, M. Macik, and L. Treml, "Reprobooktor: A concept of audiobook player for visually impaired older adults," in 2018 9th IEEE International Conference on Cognitive Infocommunications (CogInfoCom). IEEE, 2018. doi: 10.1109/CogInfoCom.2018.8639950 pp. $000063-000068$. [Online]. Available: https://doi.org/10.1109/ CogInfoCom.2018.8639950

[17] World Health Organization, "International classification of diseases, 11th revision," Dec 2018, https://icd.who.int/browse11/l-m/en\#/http://id.who. int/icd/entity/1103667651.

[18] Balsamiq. Balsamiq wireframes. [Online]. Available: https://balsamiq com/wireframes

[19] AxureSoftware. Prototypes, specifications, and diagrams in one tool. [Online]. Available: https://www.axure.com

[20] K. Caine, "Local standards for sample size at chi," in Proceedings of the 2016 CHI Conference on Human Factors in Computing Systems. ACM, 2016. doi: 10.1145/2858036.2858498 pp. 981-992. [Online]. Available: http://dx.doi.org/10.1145/2858036.2858498

[21] P. Bacchetti, S. G. Deeks, and J. M. McCune, "Breaking free of sample size dogma to perform innovative translational research," Science translational medicine, vol. 3 , no. 87, pp. $87 \mathrm{ps} 24-87 \mathrm{ps} 24$, 2011. doi: 10.1126/scitranslmed.3001628. [Online]. Available: http: //dx.doi.org/10.1126/scitranslmed.3001628

[22] J. Selin and M. Rossi, "The functional design method for buildings (fdm) with gamification of information models and ai help to design safer buildings," in 2018 Federated Conference on Computer Science and Information Systems (FedCSIS). IEEE, 2018. doi: 10.15439/2018F162 pp. 907-911. [Online]. Available: http://dx.doi.org/10.15439/2018F162 